COMMENTAIRE

\title{
Émotion, cognition et action motivée: une nouvelle vision de la neuroéducation
}

\author{
Gérardo Restrepo ${ }^{1 *}$
}

\begin{abstract}
RÉSUMÉ
Les relations entre émotion et cognition ont été un sujet de controverse et de discussions en psychologie et en sciences humaines depuis des temps immémoriaux. La philosophie, la psychologie, l'éducation et les neurosciences ont nourri cette controverse en examinant différentes façons de la comprendre. Les postures théoriques contemporaines considèrent que les émotions sont les médiateurs qui assurent l'interface entre le monde intérieur (la cognition) et le monde extérieur (la vie sociale) de l'individu. Cet article aborde la problématique de la relation entre émotion, cognition et action motivée sous l'angle de l'histoire de la pensée philosophique, des conceptions psychologiques et pédagogiques contemporaines, et à la lumière des études récentes en neurosciences. Nous discuterons de certaines questions portant sur cette interaction, notamment l'impact de ces concepts sur notre compréhension des fonctions exécutives du cerveau et le rôle des émotions dans notre vie. Un modèle explicatif de cette interaction est proposé à la fin de l'article où l'émotion joue un rôle de médiateur pédagogique du développement de l'enfant.
\end{abstract}

\footnotetext{
${ }^{1}$ Université de Sherbrooke, Département d'études sur l'adaptation scolaire et sociale, 2500, boul. de l'Université, Sherbrooke, Québec, Canada J1K 2R1

* Correspondance avec l'auteur : gerardo.restrepo@usherbrooke.ca
}

Pour citer cet article : Restrepo, G. (2014). Émotion, cognition et action motivée: une nouvelle vision de la neuroéducation. Neuroéducation, 3(1), 10-19. https://doi.org/10.24046/neuroed.20140301.10

Reçu le 25 juin 2013. Reçu en version révisée le $1^{\text {er }}$ novembre 2013 et le 2 avril 2014.

Accepté le 14 avril 2014. Disponible en ligne le 31 octobre 2014.

Neuroéducation, 3(1), 10-19

ISSN : 1929-1833

Tous droits réservés (C) 2014 - Association pour la recherche en neuroéducation / Association for Research in Neuroeducation 


\section{Introduction}

Les relations entre émotion et cognition ont été un sujet de controverse et de discussions en psychologie et en sciences humaines depuis des temps immémoriaux. De la Grèce antique à nos jours, la question de la relation entre émotion et cognition est passée de la métaphore philosophique (Platon et l'âme tripartite) à l'explication physiologique (théories de James-Lange et de Cannon-Bard), pour se poser finalement en termes plus spécifiquement psychologiques. En effet, l'angle sous lequel on analyse actuellement cette interaction souligne l'importance de la relation entre l'individu et son entourage. On peut remarquer l'existence d'un vaste consensus concernant le rôle des émotions dans la vie mentale et sociale des êtres humains. En d'autres mots, les émotions sont les médiateurs qui assurent l'interface entre le monde intérieur (la cognition) et le monde extérieur (la vie sociale) de l'individu (Oatley, Gerrod Parrott, Smith et Watts, 2011). Cependant, l'idée de la coexistence d'un être cognitif et d'un être émotionnel indépendant à l'intérieur de chaque individu est encore ancrée dans la plupart des théories psychologiques contemporaines (Richelle, 1993). Cette séparation entre émotion et cognition est profondément enracinée dans la culture et la pensée occidentale et a des retombées sur nos conceptions scientifiques, philosophiques et éducatives.

La signification initiale du mot émotion mettait l'accent sur l'action et le mouvement. D'un point de vue étymologique, le mot émotion est dérivé du terme motion en latin et du préfixe e «bouger hors» (Rimé, 2009). Les psychologues de la première moitié du $\mathrm{XX}^{\mathrm{e}}$ siècle se sont intéressés à la physiologie et à la valeur adaptative des émotions jusqu'à la révolution cognitive des années 1960, quand l'émotion a été assimilée à la cognition. Frijda (2005) identifie trois composantes des émotions : 1- l'évaluation, 2- la préparation à l'action et 3- la réponse. Le premier, l'appraisal est le composant cognitif des émotions, le deuxième est l'orientation des actions en lien avec le premier et finalement, le troisième comprend les réactions physiologiques et corporelles de l'émotion.

Cependant, quelques théoriciens de l'émotion se sont distanciés du cognitivisme et ont réaffirmé le rôle central des émotions dans la représentation du monde. Pour Benjafield, Smilek et Kingstone (2010), la signification du mot cognition inclut l'habileté et l'action de connaître. Cette définition implique que la connaissance découle de l'action de l'individu sur son entourage, et que la représentation du monde peut se faire sous plusieurs formes ou styles cognitifs guidés par l'émotion. Selon la théorie de l'activité, toutes les activités humaines naissent des besoins de l'individu de s'orienter vers un objet ou un but en lien avec l'environnement. Les actions humaines sont motivées par les relations que l'individu construit avec son entourage (Leontiev, 1978).

Cet article aborde la problématique de la relation entre émotion, cognition et action motivée sous l'angle de l'histoire de la pensée philosophique, des conceptions psychologiques et pédagogiques contemporaines et à la lumière des études récentes en neurosciences. Nous avons choisi cet angle d'analyse, car, au départ, l'explication des processus psychologiques et neuroscientifiques se faisait d'un point de vue philosophique. Il a fallu attendre jusqu'au XIX ${ }^{\mathrm{e}}$ siècle pour que la psychologie puisse se distancier de la philosophie et s'ériger comme discipline indépendante. Les neurosciences ont vécu, pour leur part, un développement extraordinaire pendant le XXe siècle pour nous permettre, à l'aube du XXI siècle, de réexaminer cette problématique d'une manière plus précise. Notre démarche tient donc compte des développements historiques de la relation entre émotion, cognition et action motivée et débouche sur les postulats récents de la neuroéducation. Dans les lignes qui suivent, nous allons discuter de certaines questions portant sur cette interaction, notamment l'impact de ces concepts sur notre compréhension des fonctions exécutives du cerveau et le rôle des émotions dans notre vie. Un modèle explicatif de cette interaction est proposé à la fin de l'article, selon lequel l'émotion joue un rôle de médiateur pédagogique du développement de l'enfant.

\section{2. Émotion et cognition: le point de vue de la philosophie}

Selon Oatley et al. (2011), les philosophes stoïciens ont été les premiers à étudier systématiquement la relation entre émotion et cognition comme un moyen de comprendre ses conséquences pour l'individu et la société. Dans le livre IV de la République, Platon (env. 428-327 av. J.-C.) expose la tripartition de lâme humaine. Le noûs, la raison, placée dans la tête, contrôle le comportement; le thymos, dans le cœur, est l'organe du courage, de la valeur et de la volonté. Finalement, les épithymia, dans le ventre, représentent les instincts et les passions. La raison doit soumettre les passions pour diriger la volonté vers un objectif. Nonobstant que cette métaphore soit une conception de contrôle social (lâme et la cité ont la même structure), sa signification implicite est que la raison et l'émotion doivent converger vers la volonté, c'està-dire l'action motivée (Psycho-politique, livre II). Platon divise le monde en deux, le monde sensible, une reproduction déformée de la réalité révélée par nos organes des sens, et celui des idées, lumineux et clair, accessible seulement par la raison (Fougeyrollas, 2000). Cette séparation entre émotion et cognition, voire entre corps et esprit, est une constante de l'histoire des idées de la culture occidentale. En effet, cognition et émotion ont toujours été conçues comme deux systèmes parallèles dont le premier doit toujours modérer et réguler le second (Oatley, 2011). Par exemple, ce contrôle de la cognition sur l'émotion est la base du concept neuropsychologique des fonctions exécutives (Powell et Voeller, 2004). De plus, une conception latente dans l'histoire de la philosophie consiste en la division entre une réalité tangible accessible par nos organes des sens et un monde d'idées seulement accessible par raison. Cette dernière conception a établi les bases pour la séparation corps et esprit et a amorcé le débat philosophique entre empirisme et 
rationalisme, source de l'épistémologie occidentale (Chiong, 2011).

La séparation entre corps et esprit a été liée à l'idée que nos organes des sens nous trompent ou déforment la réalité. Cette idée est née avec Platon et a pris son essor avec les postulats fondateurs de la philosophie cartésienne (Lewis et Haviland-Jones, 2000). La phrase, "Je pense, donc je suis", vérité fondatrice de la métaphysique cartésienne, est sans doute le point de départ de la philosophie moderne, mais aussi de la discussion sur la science, la méthode et la théorie de la connaissance. Descartes met au premier plan la pensée et fait valoir que les idées précèdent l'expérience sensible. Ainsi, dans son livre, Discours de la méthode, Descartes (1637) a donné lieu à une séparation entre la faculté de sentir et la faculté rationnelle en distinguant radicalement la matière (res extensa) de la pensée (res cogitans). Cette séparation conceptuelle entre corps et esprit est le point de départ du dualisme et de la théorie cartésienne. Par le biais de ces postulats, Descartes a mis en scène l'un des problèmes les plus importants de la philosophie et de la science, la relation entre le monde physique et sa représentation mentale. Le cheminement méthodique de Descartes l'a conduit à la conclusion que le corps et l'esprit forment deux substances différentes. Par conséquent, Descartes pensait que l'esprit pouvait exister sans le corps, que lâme, source de la raison, ne devait pas être objet de recherche et que la science devait se dévouer à l'étude de la res extensa, le corps (Lewis et Haviland-Jones, 2000).

La position cartésienne a été contestée par Francis Bacon, philosophe et homme de science anglais, contemporain de Descartes. Bacon est considéré comme le père de l'empirisme moderne. Son ouvrage philosophique Novum Organum, publié en 1620, qui prône l'observation exacte et l'expérimentation en science, constitue les fondements de l'empirisme scientifique et de la science moderne. À cette époque-là, les débats philosophiques se centraient sur quelques questions transcendantales. Certains phénomènes ou certaines choses peuvent-ils exister en dehors du sujet pensant ou sont-ils construits par le sujet? Les réponses à ces questions sont venues de l'empirisme classique sous l'influence de John Locke et d'Isaac Newton. Ce courant de pensée, qui s'oppose au rationalisme, a émergé pendant le $\mathrm{XVIII}^{\mathrm{e}}$ siècle. Contrairement au rationalisme, l'empirisme affirmait que la source de toute connaissance était l'action du monde extérieur sur nous. Pour l'empirisme, le sujet était la base de la connaissance du monde, et cette connaissance tenait sa légitimité de la vérification expérimentale et non pas d'une démonstration rationnelle (Lewis et Haviland-Jones, 2000).

Pendant que l'empirisme et le rationalisme discutaient de la source de la connaissance, la phénoménologie s'engageait dans l'étude systématique de l'expérience et de la conscience. Emmanuel Kant utilise les termes "noumène" pour désigner les réalités tangibles, les choses en soi, et "phénomène" pour nommer ce qui appartient au champ de l'expérience. La phénoménologie a pris son essor grâce aux travaux de psychologues du XIX ${ }^{\mathrm{e}}$ siècle comme Franz Brentano, William James et Wilhelm Wundt, qui utilisaient l'introspection comme méthode d'étude des fonctions psychiques (Chiong, 2011). Husserl (1913), fondateur de la phénoménologie, affirme que cette discipline doit transcender le champ de l'introspection et de l'étude de l'individu pour tenter de comprendre les principes universels de la logique de la pensée. Pour Husserl, l'intentionnalité est une propriété des phénomènes psychiques, car elle dirige les concepts et les représentations vers les objets ou les choses. L'intentionnalité de la conscience reflète la position de l'individu envers le monde : par exemple, j'aperçois un objet, il se souvient d'un événement. Cependant, le terme « conscience » est attribué de manière originale à John Locke (1690) qui, dans «An Essay Concerning Human Understanding » lui donne deux significations différentes : celle de la conscience phénoménale et celle de la conscience de soi. Il faut souligner que la discussion de ces concepts, amorcée par la philosophie et la phénoménologie, a été finalement reprise par la psychologie cognitive et la neuroscience.

C'est le cas, notamment, de la discussion sur la nature de la conscience. Soutenue au départ par la philosophie, celle-ci a été reprise par les neurosciences au cours des deux décennies précédentes (Edelman, 2002). Ainsi, le concept de conscience phénoménale a été utilisé pour expliquer certaines caractéristiques de la perception sensorielle et de l'attention sélective. Pour sa part, la conscience de soi a évolué en neurosciences vers le concept de self et de mémoire de travail (Block, 2005).

\section{3. Émotion et cognition: le point de vue de la psychologie cognitive}

Le concept de mémoire de travail comme composante principale des fonctions exécutives a été décrit pour la première fois par Baddeley et Hitch (1974) et défini postérieurement par Lezak (1983) comme le mécanisme de contrôle du comportement. Les fonctions exécutives ont été conçues comme un système de planification, de direction et de contrôle des actions vers un objectif. Les fonctions exécutives sont considérées comme des fonctions cognitives de niveau élevé sous le contrôle du lobe frontal (Stuss et Alexander, 2000). Cependant, c'est Luria (1973) qui a établi les bases du concept en affirmant que le lobe frontal est le responsable de l'organisation de la vie intellectuelle au moyen de la programmation, de la surveillance et de la direction des actions et du comportement.

Les fonctions exécutives ont été conçues, au départ, comme l'ensemble des fonctions cognitives supérieures qui déterminent le comportement intentionnel, organisé et dirigé vers un but (Lezak, 1983). Pourtant, le concept initial de fonctions exécutives comme mécanisme de contrôle cognitif de l'action a migré graduellement au fil des constatations 
croissantes de l'importance des émotions dans tout le processus. Selon Royall et al. (2002), le lobe frontal est la seule région corticale capable d'intégrer l'information sensorielle, interne et externe, l'émotion, la motivation et la mémoire dans une action unifiée dirigée vers un objectif (traduction libre, p. 379). Par conséquent, le rôle principal des fonctions exécutives est la programmation des actions, la régulation des émotions et l'adaptation du comportement au contexte social (Best et Miller, 2010). Le siège des fonctions exécutives se situe dans le cortex préfrontal humain. On reconnaît trois composantes de la fonction exécutive : le contrôle cognitif, le contrôle émotionnel et le contrôle motivationnel, respectivement associées à la partie latérale, orbitofrontale et médiane du cortex préfrontal (Miller et Cohen, 2001). La notion de contrôle rend compte des processus qui permettent, dans une situation donnée, la sélection de l'action appropriée, orientée vers un but. Elle est proche de celle de régulation. L'idée implicite dans ce concept est que, si nous avons le contrôle de la situation et que nous prenons les décisions appropriées, nous pouvons modérer l'intensité ou orienter la direction des émotions (Oatley et al., 2011).

Selon Miyake et al. (2000), il existe trois fonctions exécutives de base: la flexibilité mentale (shifting), la mise à jour (updating) et l'inhibition. La première permet l'adaptation aux situations changeantes du contexte, la deuxième la mise à jour des informations dans la mémoire de travail et, la troisième, l'inhibition des réponses automatiques ou routinières, mais non pertinentes pour la tâche en cours. Bref, nous devons dans toutes les activités et contextes de la vie quotidienne prendre des décisions, sélectionner les réponses appropriées au contexte et choisir entre plusieurs options. Pour Rolls (2012), les fonctions exécutives peuvent être considérées comme le substrat cognitif du libre arbitre. Damasio (1993) va au-delà de ce raisonnement. Pour lui, le cortex orbitofrontal fait partie d'un réseau cérébral complexe qui soutient la prise de décisions. Le cortex orbitofrontal permet de relier les informations factuelles de l'environnement à l'état émotionnel de l'individu pour guider son choix. Cette prise de décisions semble donc reposer en parties égales sur la raison et l'émotion. La volonté ne serait, par conséquent, qu'un algorithme cognitif nuancé par l'émotion.

\section{4. Émotion et cognition: le point de vue des sciences de l'éducation}

Les sciences de l'éducation ont séparé depuis longtemps l'émotion de la cognition. Bloom (1956) propose une taxonomie des processus cognitifs et affectifs essentiels à l'apprentissage, apparemment reliés entre eux, mais indépendants. Anderson et Krathwohl (2001) ont réalisé une révision de la taxonomie de Bloom pour l'adapter aux objectifs modernes de l'éducation. Le rappel, la compréhension, l'application et l'analyse ont été retenus comme processus cognitifs essentiels. Ces auteurs ont ajouté quatre dimensions de la connaissance : factuelle, conceptuelle, procédurale et métacognitive. Aucune référence aux processus affectifs n'a été faite. Cette séparation n'a jamais contribué à la compréhension du processus d'apprentissage de l'enfant. Pour sa part, le socioconstructivisme moderne fait référence de manière implicite aux émotions, mais, pour Vygotsky $(1934,1998)$, il est impensable de considérer l'émotion sans la cognition et vice versa :

Celui qui dès le début a séparé pensée et affect s'est ôté à jamais la possibilité d'expliquer les causes de la pensée elle-même car une analyse déterministe de la pensée suppose nécessairement la découverte des mobiles de la pensée, des besoins et des intérêts, des impulsions et des tendances qui dirigent le mouvement de la pensée dans un sens ou dans un autre. De même, celui qui a séparé la pensée de l'affect a rendu d'avance impossible l'étude de l'influence que la pensée exerce en retour sur le caractère affectif, volitif de la vie psychique, car l'analyse déterministe de la vie psychique exclut aussi bien l'attribution à la pensée d'une force magique capable de définir le comportement de l'homme par son seul système propre que la transformation de la pensée en un inutile appendice du comportement, en son ombre impuissante et vaine. (p. 61)

Ainsi, Vygotsky (Ibid.) insiste sur le fait que ces interactions sont à double sens et constituent la base de la personnalité et du comportement humain : « l'émotion n'est pas simplement la somme des sensations des réactions organiques, mais en premier lieu une tendance à agir dans une direction déterminée » (p. 134).

\section{5. Émotion et cognition: le point de vue des neurosciences}

Depuis quelques années, les neuroscientifiques ont commencé à se poser des questions qui, traditionnellement, intéressaient seulement les philosophes (Edelman, 2002). Le problème de la conscience, de la relation corps-esprit, ou de la relation émotion-cognition est le noyau central de la philosophie de l'esprit et de la neurophilosophie (Churchland, 2002). Comment le monde physique, le cerveau ou la matière peuvent-ils donner naissance aux phénomènes mentaux abstraits comme la pensée ou la conscience? Pour Chalmers (1998), la nature de la conscience et de l'interaction entre le corps et l'esprit constitue l'obstacle le plus important à notre compréhension de l'univers.

La recherche neuropsychologique des dernières décennies nous a légué l'idée que le cerveau est un organe modulaire qui présente un degré considérable de spécialisation fonctionnelle. Une vision particulièrement répandue est que certaines régions du cerveau sont plus affectives tandis que d'autres sont plus impliquées dans la cognition (Pessoa, 2008). Cependant, selon Gray, Braver et Raichie (2002), dans 
plusieurs étapes du traitement de l'information, la spécialisation disparaît et l'émotion et la cognition travaillent ensemble pour assurer le contrôle de la pensée et du comportement.

La vieille conception de l'âme tripartite de Platon a aussi eu sa contrepartie en neurosciences. Ainsi, en 1990, MacLean proposait sa conception d'un cerveau triunique constitué de trois formations évolutives différentes. La plus ancienne, le cerveau reptilien, contrôlerait nos pulsions fondamentales (faim, soif, sexualité, agressivité); la deuxième, le cortex limbique, le plus récent sur le plan évolutif, serait le responsable de l'affectivité, des soins parentaux et du sentiment d'appartenance à un groupe. La nature complexe de l'information affective pousserait le cerveau à développer un système de codage approprié, la mémoire à long terme et un mécanisme psychique pour diriger sélectivement nos actions, la motivation. Finalement, dans l'évolution apparaîtrait le néocortex, dépositaire de la raison, de la cognition et du langage symbolique; celui qui est responsable de la vie sociale. Le modèle du cerveau triunique de MacLean transpose en neurosciences le concept d'âme tripartite de Platon. Le néocortex, siège de la raison, doit dominer les pulsions et les émotions du système limbique et du cerveau reptilien pour guider l'action motivée, notre comportement.

En 1981, Roger Sperry et Michael Gazzaniga reçoivent le Prix Nobel de médecine pour leurs découvertes concernant la spécialisation fonctionnelle des hémisphères cérébraux. À partir de leurs travaux, une image généralement évoquée est que l'hémisphère gauche est le siège de la logique analytique et rationnelle tandis que l'hémisphère droit est émotionnel, intuitif et holistique. Selon Gazzaniga, le cerveau normal est «organisé en systèmes de traitements modulaires se dénombrant par centaines ou peut-être même par milliers » (1987, p. 69). Ces modules, relativement indépendants et spécialisés, analysent l'information de manière différentiée. Le langage verbal et la préférence manuelle sont tous deux gouvernés par l'hémisphère gauche chez la majorité des individus. Ce dernier serait un dispositif de traitement temporaire de l'information. À l'inverse, la spécialisation de l'hémisphère droit dans les émotions, l'attention, et les processus visuo-spatiaux ferait de lui un dispositif de traitement spatial de l'information (Habib, 1998). L'hypothèse de l'hémisphère droit comme base du traitement émotionnel a trouvé un appui dans la théorie du cerveau social de Brothers (1990). Selon cet auteur, l'hémisphère droit est un système neurocognitif spécifique, activé seulement par des stimuli sociaux. La théorie de l'esprit (Baron-Cohen, 1991) et les études d'imagerie du cerveau relatives à l'autisme (BaronCohen et al., 1999) et à l'empathie (Decety et Lamm, 2007) ont donné un support empirique à cette hypothèse.

La conscience comme phénomène de représentation personnelle a fait l'objet d'un débat et d'une controverse en neurosciences. «Une personne normale ne possède pas de mécanisme conscient unitaire dans lequel le système conscient peut accéder aux sources de tous les actes.
J'entends démontrer que le cerveau normal est organisé en modules et que la plupart de ces modules sont capables d'actions, d'humeurs et de réponses » affirme Gazzaniga (1987, p. 105). Le support empirique de ces affirmations provient d'études sur la communication interhémisphérique chez des individus dont le cerveau est divisé. Ainsi, Gazzaniga décrit l'apraxie diagonistique, un état causé par la section du corps calleux, qui brise la conscience corporelle unitaire. Le sujet construit une tour de cubes avec la main droite, contrôlée par l'hémisphère gauche tandis que la main gauche, contrôlée par l'hémisphère droit, essaie de construire une figure différente. D'autres observations neurologiques permettent aussi de constater la modularité cérébrale de la conscience. Par exemple, les personnes paralysées d'un côté du corps à la suite d'un accident cérébral vasculaire éprouvent une dissociation de la motricité volontaire et involontaire. Ils ne peuvent pas sourire de façon volontaire (voie pyramidale), mais ils peuvent le faire involontairement en regardant une scène comique (voie extrapyramidale). Ce phénomène montre la séparabilité de la composante cognitive (pyramidale) et émotionnelle (extrapyramidale) du mouvement (Cytowic, 1996).

En dépit du fait que plusieurs neuroscientifiques (par exemple, LeDoux, 2005; Swanson, 2003) considèrent l'émotion et la cognition comme deux systèmes séparés, divers travaux scientifiques indiquent qu'elles s'intégreraient plutôt dans l'action motivée au moyen de nombreuses connexions présentes dans le cortex préfrontal (Gray et al., 2002). La région latérale est impliquée dans un réseau qui permet l'organisation de processus cognitifs complexes tels que la planification et le raisonnement déductif; les régions orbitaires dans les processus affectifs et motivationnels; et les régions médianes dans l'autogénération des comportements et la régulation des actions (Koechlin et Summerfield, 2007). La théorie du «modèle en cascade du contrôle cognitif » de Koechlin et Summerfield (Ibid.) avance que des aires distinctes au sein du cortex préfrontal latéral intègrent l'information à différentes échelles temporelles. Selon cette théorie, les stimuli se perçoivent en lien étroit avec les événements du contexte. Ils sont ensuite comparés aux informations gardées en mémoire pour leur accorder une importance relative. Le cortex préfrontal a aussi été associé à la mémoire de travail, une mémoire à court terme où l'information est brièvement stockée pour être utilisée rapidement. La mémoire de travail traite l'information transitoire permettant à l'individu des opérations cognitives telles que le raisonnement, la compréhension, la résolution de problèmes ou encore la prise de décisions (Levy et Goldman-Rakic, 2000). Selon la théorie des marqueurs somatiques de Damasio (1995), le cortex orbitofrontal est une structure clé dans le traitement émotionnel, dans la prise de décision et dans la mémorisation des souvenirs liés aux récompenses et punitions. Le cortex orbitofrontal reçoit de multiples connexions de plusieurs systèmes sensoriels et des structures limbiques impliquées dans le traitement des émotions. Ensuite, cette région corticale associe les 
sensations émotionnelles, lesquelles sont implicites et automatiques, aux stimuli de l'environnement. Cette association sera enregistrée dans la mémoire à long terme et la personne sera en mesure de réactiver les sensations émotionnelles lors d'une rencontre ultérieure avec le stimulus concerné. Les liens entre le cortex orbitofrontal et le comportement moral et social ont aussi fait l'objet d'études par Damasio et ses collaborateurs (Koenigs et al., 2007; Immordino-Yang, McColl, Damasio et Damasio, 2009). Ainsi, le jugement et le comportement moral seraient le résultat de l'évaluation cognitive que le sujet fait des émotions partagées avec autrui (Moll, Oliveira-Souza, Bramati et Grafman, 2002). Selon Haidt (2003), les émotions morales seraient intrinsèquement liées aux intérêts de bien-être de chaque société et de chaque individu.

Par ailleurs, le cortex préfrontal médian et le cortex cingulaire (CC) antérieur jouent un rôle important dans l'établissement de liens d'empathie et de sympathie (Etkin, Egner et Kalisch, 2011). L'empathie peut se définir comme la capacité à ressentir et à comprendre les émotions et les sentiments d'autrui (Decety, 2010). Elle implique donc un partage affectif, mais aussi une compréhension minimale des états mentaux et affectifs d'autrui. La sympathie, pour sa part, permet au sujet de s'identifier avec les émotions exprimées par les autres (Ibid.). Empathie et sympathie sont des composantes essentielles du comportement prosocial, du jugement moral et de la régulation de l'agression (Ibid.). Le cortex cingulaire est aussi impliqué dans le contrôle des fonctions exécutives (CC antérieur) et dans l'évaluation des résultats des actions (CC postérieur) (Posner, Rueda et Kanske, 2007). On sait que cette région joue un rôle déterminant dans l'intégration des composantes cognitives et émotionnelles des stimuli pour assurer l'action motivée dirigée vers un objectif (Ollat, 2004). Toutefois, selon Gray et al. (2002), le processus d'intégration des émotions et de la cognition se fait de façon progressive dans plusieurs structures du cerveau. L'intégration permet à ces deux sousfonctions, séparées au départ, d'émerger pour bâtir une fonction plus complexe et générale. Un tel degré de précision et de complexité s'expliquerait par le fait que les composantes cognitive et émotionnelle détiennent la même importance dans l'organisation du cortex préfrontal (Gray et al., 2002). Les résultats obtenus par ces auteurs soutiennent l'idée que l'intégration de l'information cognitive et émotionnelle s'avère un facteur crucial pour le contrôle unifié de la pensée, de l'affectivité, de la motivation et du comportement. Une intégration fonctionnelle des émotions et de la cognition devrait permettre, selon Gray et al. (2002) au comportement d'être dirigé vers un objectif, c'est à dire permettre l'action motivée.

Dans les paragraphes précédents, nous avons souligné l'importance du cortex préfrontal dans le processus d'intégration des émotions et de la cognition. Le cortex préfrontal latéral sélectionne et catégorise l'information cognitive importante en lien avec le contexte. Le cortex orbitofrontal reçoit l'information émotionnelle du corps et l'associe à l'environnement physique et social où se déroule l'activité. Le cortex cingulaire intègre émotion et cognition pour assurer l'action motivée, en tenant toujours compte du jugement moral et de l'évaluation empathique de l'action. Les connaissances actuelles des neurosciences nous donnent ainsi la possibilité de comprendre la relation intime entre émotion, cognition et action motivée. Reste à expliquer, cependant, l'imbrication graduelle du processus au cours du développement de l'enfant.

\section{Cognition, émotion et action motivée: vers une intégration globale}

Pour Wallon (1938, dans Jalley, 1998), la vie émotionnelle est le «premier terrain des relations interindividuelles de conscience » et le «point de départ de la vie représentative (p. 39) ». Cet auteur affirme aussi que « La racine de la conscience, donc de la représentation, est à chercher non dans l'activité extéroceptive, mais dans l'activité proprioceptive » (Ibid., p. 72). Craig (2002) et Damasio (1993) vont dans le même sens que Wallon en identifiant émotions, information proprioceptive et conscience. Les sensations somatiques, porteuses des informations extéroceptives, de l'audition et de la vision, sont associées aux informations proprioceptives qui rendent compte de l'état émotionnel de l'individu. La conscience phénoménale est nourrie par l'information extéroceptive, tandis que la conscience d'accès est supportée par l'émotion, l'information qui vient du corps. Selon Cornelius (2006), les émotions sont centrales dans la relation des êtres humains avec l'environnement et cette relation explique pourquoi chaque émotion est évaluée en relation avec l'environnement social du sujet. Les émotions sont donc les médiateurs qui assurent l'interface entre le monde intérieur (la cognition) et le monde extérieur (la vie sociale) de l'individu (Oatley, 2011).

Le rôle de médiation des émotions et l'intégration progressive de l'émotion et de la cognition dans plusieurs structures du cerveau nous amènent graduellement aux vieux problèmes de la philosophie. Ainsi, peu importe la façon dont on comprend le concept de fonction exécutive, celui-ci nous amène, de façon incontournable, au problème de la conscience et de la volonté. Les travaux de Block (2005) aident à mieux comprendre la nature du phénomène. En effet, l'auteur reprend à la lumière des neurosciences la vieille discussion phénoménologique concernant la volonté, l'intentionnalité et la conscience, et s'intéresse aux opérations cognitives et neurales qui permettent d'amener une information à la conscience. Il propose l'existence de deux corrélats neuraux de la conscience: la conscience phénoménale et la conscience d'accès. La conscience phénoménale est la perception des objets ou des formes, une expérience subjective et riche, mais surtout personnelle et unique. Ces phénomènes, désignés par Lewis (1929) sous le terme de qualia, sont les qualités particulières de l'expérience sensible ou «phénoménale ». La conscience phénoménale 
précède et déclenche l'activité cognitive, amenant le sujet, ce faisant, vers un objectif par le biais de la conscience d'accès. Posner, Rueda et Kanske (2007) distinguent les processus mentaux automatiques de ceux non automatiques pour expliquer le fonctionnement du réseau attentionnel. La conscience d'accès serait donc un système de contrôle volontaire, hiérarchiquement organisé, ayant pour objectif de diriger la cognition et le comportement (Shallice, 1982). Étant donné que la conscience d'accès effectue le traitement simultané de plusieurs types d'information, il est possible de la comparer, dans une certaine mesure, à la mémoire de travail qui, après avoir reçu l'information sensorielle de plusieurs sources distinctes, sélectionne l'information et la traite en fonction d'un objectif donné. Les neuroscientifiques semblent de cette façon partager la notion de Husserl (1913), selon laquelle l'élément central de la conscience est l'intentionnalité, c'est-à-dire la conscience comme processus perceptuel et cognitif, dirigée vers l'individu, ou la conscience comme action intentionnelle dirigée vers un objectif externe.

C'est ce qui nous amène à parler d'une nouvelle discipline, la neuroéducation, laquelle ouvre la voie à l'opérationnalisation de ces concepts en enrichissant la pratique pédagogique.

\section{7. Émotion, cognition et action motivée: la vision de la neuroéducation}

La neuroéducation est une jeune discipline qui prône une vision transdisciplinaire de l'apprentissage et du développement de l'enfant (Battro, Fischer et Léna, 2008). Comme discipline émergente, selon Bruer (2007), son principal défi est la construction de liens entre la neurobiologie et les sciences de l'apprentissage. Intégrer les connaissances des neurosciences, la pédagogie et la psychologie du développement pour reconnaître l'enfant comme un acteur productif de son apprentissage et non pas comme un récepteur passif d'information (Levi-Montalcini, 2007). La psychologie et les neurosciences ont exploré exhaustivement les liens entre émotion et cognition sous l'hypothèse que la cognition doit contrôler et réguler l'émotion (Oatley, 2011). Cependant, les données de la recherche neuropsychologique suggèrent que les deux systèmes ne sont pas subordonnés, mais surtout consensuels. Dans les paragraphes suivants, nous allons proposer un modèle pour l'intégration conceptuelle de ces recherches en éducation.

Les découvertes récentes des neurosciences permettent de comprendre la dynamique de l'interaction entre émotion, cognition et action motivée d'une manière holistique et novatrice. À la lumière de ces constats, émotion et cognition sont deux éléments indissociables de la vie et de l'activité humaine. Émotion et cognition s'unissent pour former l'action motivée. L'émotion ne doit plus jamais être conçue comme système subordonné de la cognition. Elle joue un rôle de médiateur du développement de l'enfant. L'impact que les recherches des neurosciences ont eu sur les théories du développement et la pédagogie est remarquable (Bruer, 2007). Il est bien connu que les chercheurs qui s'intéressent depuis plusieurs décennies au développement de l'enfant sont divisés entre les théoriciens qui pensent que le développement est le résultat de la génétique et de la biologie et ceux qui considèrent que l'environnement est la base fondamentale de l'apprentissage. Apprentissage et développement étaient donc conçus jusqu'à maintenant comme deux phénomènes indépendants et sans connexion. De fait, l'une des théories du développement de l'enfant les plus influentes aujourd'hui, la théorie piagétienne, n'a abordé les effets de l'apprentissage sur le développement cognitif de manière systématique qu'à la fin des années 1980 (Carretero, 1996). Cependant, pour la neuroéducation, l'apprentissage et le développement sont également importants, mais l'interaction entre eux permet à l'apprentissage de provoquer et de guider le développement (Bruer, 2007). Cet aspect essentiel de la neuroéducation la rapproche de la théorie socioconstructiviste, car pour cette théorie les processus évolutifs ne coïncident pas avec les processus d'apprentissage (Battro et al., 2008). Les périodes critiques sont réelles, mais leur existence ne nous permet pas de penser que la clé de l'apprentissage et du développement se trouve dans la biologie. C'est plutôt la dynamique de l'interaction entre l'individu et son environnement qui constitue la base de l'apprentissage et du développement. Cette nouvelle conception de l'interaction entre apprentissage et développement, de nature dialectique et dynamique, permet une vision holistique des composantes biologiques et culturelles du développement de l'enfant. De la même manière, la reconnaissance de cette dynamique revitalise le rôle de l'enseignement dans les difficultés de développement de l'enfant (Immordino-Yang et al., 2009). L'émotion est donc le noyau central qui assure la communication entre la vie psychique et la vie sociale. L'apprentissage et le développement de l'enfant dépendent d'un délicat équilibre entre ces deux composantes. Reconnaître cet équilibre et guider l'apprentissage avec intelligence, subtilité, respect et avec le soutien affectif approprié se révèlent des qualités essentielles d'un enseignement efficace. De la même façon que les neurosciences nous ont permis de regarder les vieux paradigmes de la philosophie sous un angle différent, nous sommes convaincus qu'elles pourront nous guider dans la construction d'une nouvelle science de l'apprentissage.

\section{Références}

Anderson, L. W. et Krathwohl, D. (dir.). (2001). A taxonomy for learning, teaching, and assessing: A revision of Bloom's taxonomy of educational objectives. New York, NY: Longman. 
Baddeley, A. et Hitch, G. (1974). Working memory. Dans G. H. Bower (dir.), Psychology of Learning and Motivation (vol. 8) (p. 47-89). New York, NY: Academic Press. https://doi.org/10.1016/s0079-7421(08)60452-1

Baron-Cohen, S. (1991). The development of a theory of mind in autism: Deviance and delay? Psychiatric Clinics of North America, 14(1), 33-51.

Baron-Cohen S., Ring H. A., Wheelwright S., Bullmore E. T., Brammer M. J., Simmons A. et Williams S. C. (1999). Social intelligence in the normal and autistic brain: an fMRI study. European Journal of Neurosciences, 11(6), 1891-1898. https://doi.org/10.1046/j.1460-9568.1999.00621.x

Battro, A. M., Fischer, K. W. et Léna, P. J. (dir.). (2008). The educated brain. Cambridge, Royaume-Uni: Cambridge University Press. https://doi.org/10.1017/cbo9780511489907

Benjafield, J. G., Smilek, D. et Kingstone, A. (2010). Cognition (4e édition). Don Mills, ON: Oxford University Press.

Best J. R. et Miller P. H. (2010). A developmental perspective on executive function. Child Development, 81(6), 16411660. https://doi.org/10.1111/j.1467-8624.2010.01499.x

Block, N. (2005). Two neural correlates of consciousness. Trends in Cognitive Sciences, 9(2), 46-52. https://doi.org/10.1016/j.tics.2004.12.006

Bloom, B. (dir.). (1956). Taxonomy of educational objectives: The classification of educational goals. Handbook I, Cognitive Domain. New York, NY: Longman.

Brothers, L. (1990). The social brain: A project for integrating primate behavior and neurophysiology in a new domain. Concepts in NeuroScience, 1, 27-51.

Bruer, J. (2007). Building bridges in neuroeducation. Dans A. Battro, K. Fischer et P. Léna (dir.), The educated brain: Essays in neuroeducation (p. 43-58). Cambridge, Royaume-Uni: Cambridge University Press. https://doi.org/10.1017/cbo9780511489907.005

Carretero, M. (1996). Introducción a la psicología cognitiva. Buenos Aires, Argentine : Aique.

Chalmers, D. (1998). The problems of consciousness. Dans H. Jasper, L. Descarries, V. Castellucci, et S. Rossignol (dir.), Consciousness: At the Frontiers of Neuroscience. Advances in Neurology (Volume 77). Philadelphie, PA: LippincottRaven Publishers.

Chiong W. (2011). The self: From philosophy to cognitive neuroscience. Neurocase, 17(3), 190-200. https://doi.org/10.1080/13554794.2010.532808

Churchland, P. S. (2002). Brain-wise: Studies in Neurophilosophy. Cambridge, MA: MIT Press.
Cornelius, R. (2006). Magda Arnold's Thomistic theory of emotion, the self-ideal, and the moral dimension of appraisal. Cognition and Emotion, 20(7), 976-1000. https://doi.org/10.1080/02699930600616411

Craig, A. B. (2002). How do you feel? Interoception: The sense of the physiological condition of the body. Nature Reviews Neuroscience, 3(8), 655-666. https://doi.org/10.1038/nrn894

Cytowic, R. E. (1996). The neurological side of neurology. Cambridge, MA: MIT Press.

Damasio A. R. (1995). On some functions of the human prefrontal cortex. Annals of the New York Academy of Sciences, 769(1), 241-252. https://doi.org/10.1111/j.17496632.1995.tb38142.x

Damasio, A. R. (1993). Descartes' error: Emotion, reason and the human brain. New York, NY: Putnam.

Damasio, A. R., Everitt, B. J. et Bishop, D. (1996). The somatic marker hypothesis and the possible functions of the prefrontal cortex. Philosophical Transactions of the Royal Society of London. Series B : Biological Sciences, 351(1346), 1413-1420. https://doi.org/10.1098/rstb.1996.0125

Descartes, R. (1637). Discours de la méthode. Version numérique par J. M. Tremblay. En ligne: http://classiques.uqac.ca/classiques/Descartes/disco urs_methode/discours_methode.html

Decety J. (2010). The neurodevelopment of empathy in humans. Developmental Neuroscience, 32(4), 257-267. https://doi.org/10.1159/000317771

Decety, J., et Lamm, C. (2007). The role of the right temporoparietal junction in social interaction: How lowlevel computational processes contribute to metacognition. The Neuroscientist, 13(6), 580-593. https://doi.org/10.1177/1073858407304654

Decety, J. et Michalska, K. J. (2010). Neurodevelopmental changes in the circuits underlying empathy and sympathy from childhood to adulthood. Developmental Science, 13(6), 886-899. https://doi.org/10.1111/j.14677687.2009.00940.x

Edelman, G. M. (2002). Displacing metaphysics: Consciousness research and the future of neuroscience. Dans F. Mora (dir.), Continuum: Cómo funciona el cerebro (p. 147-166). Madrid, Espagne: Alianza Ensayo.

Etkin A., Egner T. et Kalisch, R. (2011). Emotional processing in anterior cingulate and medial prefrontal cortex. Trends in Cognitive Sciences, 15(2) 85-93. https://doi.org/10.1016/j.tics.2010.11.004

Fougeyrollas, P. (2000). Métamorphoses de la philosophie. Platon, Descartes, Kant, Nietzsche. Paris, France: L'Harmattan. 
Frijda, N. H. (2005). Emotion experience. Cognition \& Emotion, 19(4), 473-498. https://doi.org/10.1080/02699930441000346

Gazzaniga, M. S. (1987). Le cerveau social (trad. J. Polanis), Paris, France : Laffont.

Gray, J. R., Braver, T. S. et Raichle, M. E. (2002). Integration of emotion and cognition in the lateral prefrontal cortex. Proceedings of the National Academy of Sciences, 99(6), 4115-4120. https://doi.org/10.1073/pnas.062381899

Habib, M. (1998, mai). Latéralisation hémisphérique des émotions: Approches expérimentales et débats théoriques. Dans M. Habib et S. Bakchine (dir.), Neurologie des émotions et de la motivation. Rapport du LXXXXVI ${ }^{e}$ Congrès de psychiatrie et neurologie de langue française. La Réunion, France.

Haidt, J. (2003). The moral emotions. Dans R. J. Davidson, K. R. Scherer et H. H. Goldsmith (dir.), Handbook of affective sciences (p. 852-870). Oxford, Royaume-Uni: Oxford University Press.

Husserl, E. (1913). Ideas: General introduction to pure phenomenology. (Trad. W. R. Boyce Gibson). Londres, Royaume-Uni: George Allen et Unwin Ltd (éd. 1931).

Immordino-Yang M. H., McColl A., Damasio H. et Damasio A. (2009). Neural correlates of admiration and compassion. National Academy of Sciences. 106(19), 8021-8026. https://doi.org/10.1073/pnas.0810363106

Jalley, E. (1998). Freud, Wallon, Lacan - L'enfant au miroir. Paris, France : EPEL.

Koechlin, E. et Summerfield, C. (2007). An information theoretical approach to prefrontal executive function. Trends in Cognitive Sciences, 11(6), 229-235.

https://doi.org/10.1016/j.tics.2007.04.005

Koenigs, M., Young, L., Adolphs, R., Tranel, D., Cushman, F., Hauser, M. et Damasio, A. (2007). Damage to the prefrontal cortex increases utilitarian moral judgements. Nature, 446(7138), 908-911.

https://doi.org/10.1038/nature05631

Krathwohl, D., Bloom, B., et Masia, B. (1956). Taxonomy of educational objectives. Handbook II: Affective Domain. New York, NY: David McKay.

LeDoux J. (2005). Le cerveau des émotions. Paris, France : Odile Jacob.

Leontiev, A. N. (1978). Activity, consciousness and personality. Englewood Cliffs, NJ: Prentice-Hall.
Levi-Montalcini, R. (2007). Foreword: Towards a new pedagogical and didactic approach. Dans A. Battro, K. Fischer et P. Léna (dir.), The educated brain: Essays in neuroeducation (p. xxi-xxiv). Cambridge, Royaume-Uni et New York, NY: Cambridge University Press. https://doi.org/10.1017/cbo9780511489907.002

Levy, R. et Goldman-Rakic, P. S. (2000). Segregation of working memory functions within the dorsolateral prefrontal cortex. Experimental Brain Research, 133(1), 23-32. https://doi.org/10.1007/s002210000397

Lewis, C. I. (1929). Mind and the world-order: outline of a theory of knowledge. New York, NY: Dover.

Lewis, M. et Haviland-Jones, J. M. (dir.). (2000). Handbook of emotions ( $2^{\mathrm{e}}$ edition). New York, NY: Guilford.

Lezak, M. D. (1983). Neuropsychological assessment $\left(2^{\mathrm{e}}\right.$ édition). New York, NY: Oxford University Press.

Luria, A. R. (1973). The working brain: An introduction to neuropsychology. New York, NY: Basic.

MacLean, P. D. (1990). The triune brain in evolution. New York, NY: Plenum Press.

Miller, E. K. et Cohen, J. D. (2001). An integrative theory of prefrontal cortex function. Annual Review of Neuroscience, 24(1), 167-202. https://doi.org/10.1146/annurev.neuro.24.1.167

Miyake, A., Friedman, N. P., Emerson, M. J., Witzki, A. H., Howerter, A., et Wager, T. D. (2000). The unity and diversity of executive functions and their contributions to complex "frontal lobe" tasks: A latent variable analysis. Cognitive Psychology, 41(1), 49-100. https://doi.org/10.1006/cogp.1999.0734

Moll, J., Oliveira-Souza, R., Bramati, I. E., et Grafman, J. (2002). Functional networks in emotional moral and nonmoral social judgment. Neuroimage, 16(3), 696-703. https://doi.org/10.1006/nimg.2002.1118

Oatley, K., Gerrod Parrott, W., Smith, C., et Watts, F. (2011). Cognition and emotion over twenty-five years. Cognition and Emotion, 25(8), 1341-1348. https://doi.org/10.1080/02699931.2011.622949

Ollat H. (2004). Le cortex cingulaire antérieur à l'interface de l'émotion et de la cognition. Les données de la neuroimagerie. NPTD, 23, 37-41.

Pessoa L. (2008). On the relationship between emotion and cognition. National Review of Neuroscience, 9(2), 148-158. https://doi.org/10.1038/nrn2317

Posner, M. I. et Rothbart, M. K. (2007). Research on attention networks as a model for the integration of psychological science. Annual Review of Psychology, 58(1), 1-23. https://doi.org/10.1146/annurev.psych.58.110405.08551 $\underline{6}$ 
Posner, M. I., Rueda, M. R. et Kanske, P. (2007). Probing the mechanisms of attention. Dans J. T. Cacioppo, J. G. Tassinary et G. G. Berntson (dir.), Handbook of Psychophysiology (3e edition) (p. 410-432). Cambridge, Royaume-Uni: Cambridge University Press. https://doi.org/10.1017/cbo9780511546396.018

Powell, K. B. et Voeller, K. S. (2004). Prefrontal executive function syndromes in children. Journal of Child Neurology, 19(10), 785-797. https://doi.org/10.1177/08830738040190100801

Richelle, M. (1993). Du nouveau sur l'esprit? Paris, France: PUF. https://doi.org/10.3917/puf.riche.1993.01

Rimé, B. (2009). Le partage social des émotions. Paris, France: PUF. https://doi.org/10.3917/puf.mosco.2009.01

Rolls E. T. (2012). Willed action, free will, and the stochastic neurodynamics of decision-making. Frontiers in Integrative Neuroscience, $6,68$.

https://doi.org/10.3389/fnint.2012.00068

Royall, D. R., Lauterbach, E. C., Cummings, J. L., Reeve, A., Rummans, T. A., Kaufer, D. I., ... et Coffey, C. E. (2002). Executive control function. A review of its promise and challenges for clinical research. A report from the Committee on Research of the American Neuropsychiatric Association. The Journal of Neuropsychiatry and Clinical Neurosciences, 14(4), 377405. https://doi.org/10.1176/jnp.14.4.377

Shallice, T. (1982). Specific impairments of planning. Philosophical Transactions of the Royal Society of London. Series B, Biological Sciences, 298(1089), 199-209. https://doi.org/10.1098/rstb.1982.0082

Stuss, D. T. et Alexander, M. (2000). Executive functions and the frontal lobes: A conceptual view. Psychological Research, 63(3-4), 289-298. https://doi.org/10.1007/s004269900007

Swanson, L.W. (2003). Brain Architecture. Oxford, RoyaumeUni: Oxford University Press.

https://doi.org/10.1093/med/9780195378580.001.0001

Vygotsky, L. S. (1998). Théorie des émotions. Étude historicopsychologique (trad. N. Zavialoff, C. Saunier). Paris, France: L'Harmattan.

Vygotsky, L. S. (1934/1985). Pensée et langage (trad. F. Sève). Paris, France: Messidor. 